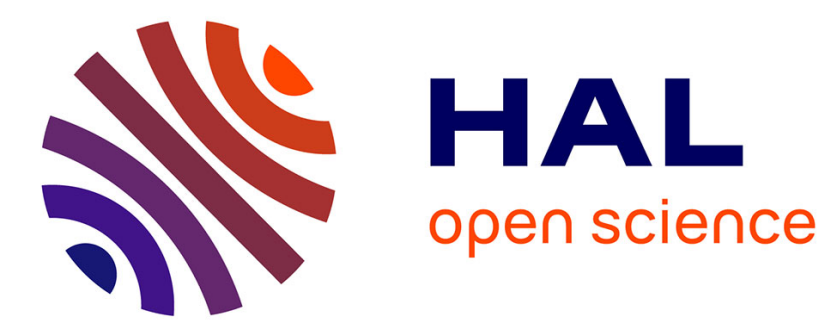

\title{
Réflexions énonciatives autour de "maintenant" argumentatif
}

Sylvie Mellet

\section{To cite this version:}

Sylvie Mellet. Réflexions énonciatives autour de "maintenant" argumentatif. Cahiers Chronos, 2008, 20, pp.77-92. hal-00552942

\section{HAL Id: hal-00552942 \\ https://hal.science/hal-00552942}

Submitted on 6 Jan 2011

HAL is a multi-disciplinary open access archive for the deposit and dissemination of scientific research documents, whether they are published or not. The documents may come from teaching and research institutions in France or abroad, or from public or private research centers.
L'archive ouverte pluridisciplinaire HAL, est destinée au dépôt et à la diffusion de documents scientifiques de niveau recherche, publiés ou non, émanant des établissements d'enseignement et de recherche français ou étrangers, des laboratoires publics ou privés. 


\title{
Réflexions énonciatives autour de maintenant argumentatif
}

\author{
Sylvie MELLET \\ CNRS, UMR 6039 ‘Bases, Corpus et Langage’, Nice
}

\section{Introduction}

L'adverbe maintenant passe pour le prototype de l'adverbe temporel déictique, du moins si on en juge par sa place dans la terminologie métalinguistique, que ce soit sous sa forme française (le « ici et maintenant » de l'énonciation), latine (les trois coordonnées déictiques de l'énonciation «ego, hic et nunc ») ou sa forme grecque (la relation nynégocentrique de Damourette et Pichon) ${ }^{1}$. Mais cet adverbe connaît aussi des emplois particuliers qui paraissent l'éloigner de sa valeur déictique fondamentale et que l'on peut qualifier, en première approximation, d'argumentatifs.

En réalité, il s'agit d'une série d'occurrences assez variées, qui présentent des points communs (notamment la position détachée à l'initiale de phrase), mais aussi des différences assez sensibles aux niveaux sémantique et fonctionnel, et qui forment ainsi un continuum énonciativoargumentatif méritant d'être examiné de plus près. L'analyse de ces différents emplois se fera sur des exemples attestés en corpus ${ }^{2}$, avec des contextes assez larges pour permettre la mise en évidence et la caractérisation de quelques facteurs contextuels déterminants dans l'interprétation de l'adverbe. Je tenterai ensuite de mettre au jour la fonction centrale de maintenant, celle qui assure la stabilité de son signifié à travers la diversité de ses emplois, ce qui devrait nous permettre d'établir aussi un lien avec ses emplois comme déictique temporel et de retrouver l'unité de sa valeur sémantique et des opérations énonciatives dont il est la trace en discours. peu sommaire ; voir en particulier Adam (1987).

2 La majorité d'entre eux sont empruntés à FRANTEXT. D’autres m’ont été aimablement fournis par Marcel Vuillaume que je remercie chaleureusement. 


\section{Les emplois de maintenant en position détachée initiale : une palette aux nuances variées}

1.1. En tant que simple adverbe temporel, maintenant est très souple d'emploi, ce qui lui permet de prendre en charge les différentes portées susceptibles d'incomber à un adverbe ;

- il peut être un modifieur de constituant à portée syntagmatique, donc infrapropositionnelle ; dans cette fonction, il ne peut évidemment pas être placé en tête d'énoncé :

(1) $[\ldots]$ cette vie maintenant si fragile (ex. fabriqué)

- mais il peut aussi définir l'intervalle de validité du prédicat ; il a alors une portée propositionnelle et, à ce titre, peut être placé sous le focus d'une interrogation, d'une négation ou d'un détachement à gauche :

(2) C'est maintenant que tu te décides ? (ex. fabr.)

(3) Il a souffert toute la journée ; maintenant, grâce à la morphine, il dort enfin d'un sommeil presque paisible. (id.)

- il peut enfin être adverbe de phrase et porter non pas sur le contenu de celle-ci, mais sur l'acte énonciatif qui la profère ou sur l'étape discursive à laquelle le locuteur invite son interlocuteur :

(4) Je partis aussitôt, le fusil sur l'épaule, en allongeant le pas du côté de la porte neuve. Et maintenant si je vous racontais l'horreur du massacre dans ce quartier, vous auriez de la peine à me croire. (E. Erckmann - A. Chatrian, Histoire d'un paysan)

(5) [...] ensemble elles composent un ange de pierre. Et d'autres, ensemble, composent une ogive. Et d'autres ensemble une colonne. Et maintenant si tu prends ces anges de pierre, ces ogives et ces colonnes, tous ensemble composent un temple. Et maintenant si tu prends tous les temples, ils composent la ville sainte. (A. de Saint Exupéry, Citadelle)

Dans ces deux derniers emplois, maintenant peut donc occuper la première place dans l'énoncé ; il reste très nettement adverbe temporel, tant par son signifié que par sa compatibilité avec un coordonnant, mais on pressent que cette fonction s'enrichit déjà de celle de ponctuant énonciatif ${ }^{3}$.

3 Selon F. Nef, même en emploi propositionnel l'adverbe de temps porte sur l'énonciation, rapportant et donc relativisant la vérité de la proposition au moment même de son énonciation (1983: 360). Cette "dérive naturelle » (Damourette \& Pichon) s'observe pou un certain nombre d'autres adverbes : sur ce point, voir en particulier Jaubert (1993). 
1.2. Lorsqu'il soutient et structure la progression discursive, maintenant se charge à l'évidence d'une fonction cohésive ; on peut alors lui donner le statut de connecteur - statut assez large pour couvrir diverses nuances d'emploi ;

- maintenant accompagne volontiers un changement d'espace de référence explicité par l'emploi de ce que Michel Charolles appelle un "cadratif», c'est-à-dire une expression plus ou moins développée qui cadre, qui indexe une ou plusieurs propositions subséquentes. Sans être lui-même à proprement parler un cadratif, maintenant participe de cette relation d'indexation, fournissant une sorte de balise organisatrice du discours ; il articule alors non pas deux énoncés, mais deux séquences plus ou moins longues :

On voit qu'en représentant la terre par 1, Jupiter, par exemple, a un diamètre 11 fois plus grand, et Mercure un diamètre qui n'est que les 37 centièmes, ou un peu moins des 4 dixièmes du nôtre. [...]. Le premier de ces deux tableaux nous montre qu'en représentant par 1 la distance de la terre au soleil, celle de Mercure est désignée par les 387 millièmes, c'est-àdire que Mercure est à un peu plus du tiers de la distance du soleil à la terre, en partant du soleil, Vénus aux 7 dixièmes environ, Mars une fois et demie plus loin que nous, Jupiter 5 fois plus loin, et ainsi de suite. Maintenant, au point de vue de l'absolu, comme ce n'est pas la terre, mais le soleil, qui est le centre de comparaison et le régulateur, il sera intéressant pour nous de nous représenter les distances des planètes exprimées en proportions du diamètre du soleil, les volumes et les masses en proportions du volume et de la masse de cet astre, et ce nouveau tableau sera plus naturel que les premiers, puisque le soleil est la véritable unité sidérale de notre système, à laquelle tout doit être rapporté. (C. Flammarion, Astronomie populaire) [passage d'un point de vue relatif centré sur la terre à un point de vue absolu centré sur le soleil]

(7) Elle était repartie. Ils surent, dit-on, que là ousqu'elle s'en était allée, c’était à la hutte de ce chef qu'ils s'y étaient unis, eux deux. Maintenant pour ce qui est de lui, à son tour le chef fut apparemment surpris de la voir de retour. (Aragon, Euvre poétique tome 1, livre 3)

- dans ce rôle structurant, maintenant connaît parfois des emplois de type métadiscursif par lesquels il confirme la clôture d'un acte illocutoire précédent et ouvre une nouvelle étape discursive :

(8) Voyons, vous êtes sûr que je ne peux pas perdre cette somme, qu'il n'est pas possible de ne pas vendre 7000 exemplaires ? Moi aussi. Maintenant vous est-il possible, avez-vous le temps, la patience, la volonté, de vous mettre à la tête d'une affaire qui nécessite peut-être plus de soins, de peines, de surveillance que [...]. (G. Sand, Correspondance) [citation qu'on pourrait gloser par: "Je vous ai communiqué ma certitude que l'affaire est prometteuse et rapportera de quoi couvrir nos investissements; mais 
maintenant j'ajoute une réserve en exprimant mon incertitude sur votre capacité à attendre ces fruits »; on reviendra plus bas sur ce type de glose]

Que Bocage paye ses fautes, c'est trop juste, et Claudie les a largement payées d'avance. Maintenant, pour contenter tout le monde, voici ce qu'on pourrait peut-être faire. - Vous diriez au directeur du Gymnase : « ...» (G. Sand, Correspondance) [Ici, on passe d'une assertion ferme qui semble clôre le débat à une proposition nouvelle qui rouvre la discussion sous forme hypothétique ; maintenant souligne qu'on a affaire à une nouvelle étape discursive, voici marque le surgissement ex nihilo de la nouvelle proposition]

Ce type d'emploi est particulièrement fréquent dans la correspondance de Georges Sand, qui recourt par ailleurs volontiers à d'autres expressions métadiscursives dialogiques telles que «je dois vous avouer », «je crois vous avoir dit », « je tiens à vous dire », etc. ${ }^{4}$ L'emploi de maintenant, qu'on peut facilement gloser par l'expression cela dit qui en est, dans ce contexte, un synonyme presque parfait ${ }^{5}$, paraît relever de la même attitude énonciative en contexte épistolaire : dans les deux cas, il s'agit de ponctuer la progression discursive et de solliciter par là-même l'attention du destinataire.

1.3. Enfin, maintenant à l'initiale de l'énoncé peut aussi prendre en charge la fonction d'opérateur argumentatif, ou plutôt contre-argumentatif puisqu'il collabore à l'expression d'une opposition, voire d'une concession, participant avec d'autres indices contextuels à une inversion d'orientation argumentative. Il est alors commutable avec des adverbes tels que cependant, toutefois ou en revanche :

(10) À Monsieur Gaillard. 4 janvier. Je pense, Monsieur, et j'ai toujours vu jusqu'à ce jour, qu'il y a économie et avantage à réunir tous les cartons ensemble et à en faire, selon le nombre, soit une demi-feuille, soit un quart, soit une feuille entière. C'est ce qui me faisait attendre la fin des bonnes feuilles. Maintenant si vous voulez tirer les cartons les uns après les autres, cela ira moins vite et vous coûtera plus cher. Je n'en suis pas moins prêt à livrer tous ceux que j'ai déjà notés et je les donnerai dès aujourd'hui. On peut venir les chercher. Croyez, je vous prie, à ma vive cordialité. (V. Hugo, Correspondance) [L'adverbe articule les points de vue opposés de l'écrivain et de son destinataire].

(11) Quant à ce soir, je ne compte pas la voir ; maintenant si elle le désirait ou si vous trouviez un joint, vous n'avez qu'à m'envoyer un mot chez Mme de Saint-Euverte jusqu'à minuit, et après chez moi. (M. Proust, Du côté de chez

4 A titre de comparaison, la correspondance de Flaubert ne contient presque aucune occurrence de maintenant en emploi métadiscursif.

$5 \quad$ Voir Rossari et al. (2004 : 106-117). Voir aussi la définition du TLF. 
Swann) [L'adverbe articule deux points de vue successifs et divergents du même énonciateur qui ne se résigne pas à entériner sa première hypothèse].

Par exemple, prends la question de la culpabilité. Politiquement, abstraitement, un individu qui a travaillé avec les Allemands est un salaud, on lui crache dessus, il n'y a pas de problème. Maintenant si tu en vois de près un en particulier, ce n'est plus du tout la même chose. - Tu penses à ton père ? dit Henri. - Oui ; il y a quelque temps que je voulais te demander conseil. (S. de Beauvoir, Les Mandarins) [L'adverbe articule le point de vue général et le point de vue particulier successivement adoptés par l'énonciatrice].

Bien évidemment, la distinction entre ces trois fonctions (adverbe temporel, connecteur à fonction cohésive et opérateur argumentatif) n'a de réalité que didactique, pour la clarté de l'exposé. Dans la réalité des textes, ces fonctions sont le plus souvent étroitement entremêlées et si le contexte permet en général de donner la prééminence à l'une d'entre elles, certaines occurrences offrent des exemples intéressants de plurivocité par surdétermination, c’est-à-dire par cumul des différentes valeurs possibles ${ }^{6}$ :

Henri ne bougea pas ; il lui semblait qu'on lui demandait de tuer Sézenac avec ses propres mains. - Qu'est-ce qui ne colle pas ? dit Vincent. On ne peut pas le laisser là, non? Maintenant si tu ne veux pas m'aider, ça va, prête-moi seulement la bagnole et je tâcherai de m'en tirer sans toi. (S. de Beauvoir, Les Mandarins) [superposition de la fonction de connecteur métadiscursif illocutionnaire et d'opérateur argumentatif annonçant la concession ça va].

(14) Capitaine, je hais parler de moi. Déjà plusieurs fois depuis hier je me suis fait une sorte de violence en vous expliquant les améliorations que j'ai pu obtenir ici ; mais il s'agissait de la commune et de ses habitants, aux intérêts desquels les miens se sont nécessairement mêlés. Maintenant, vous dire mon histoire, ce serait ne vous entretenir que de moi-même, et ma vie est peu intéressante. (H. de Balzac, Le médecin de campagne) [superposition de la valeur temporelle, maintenant pouvant s'opposer à plusieurs fois depuis hier, de la fonction cadrative et de la fonction argumentative, l'adverbe contribuant à opposer les deux séquences, celle qui évoque l'histoire collective de la commune et celle qui évoque l'histoire individuelle du locuteur].

Maintenant fait donc partie de la vaste famille des marqueurs polyfonctionnels. Parmi ses nombreux emplois, ceux qui nous intéressent ici ont au moins trois caractéristiques communes :

- la position détachée initiale ; 
- la mise en relation des valeurs illocutionnaires des énoncés ou des séquences connectés ;

- la portée énonciative de l'adverbe.

Ce sont ces deux derniers points que nous allons approfondir à présent.

\section{Un marqueur de rectification ou d'inversion illocutionnaire}

\subsection{Les analyses de F. Nef}

C'est, à notre connaissance, F. Nef qui, le premier, dans sa thèse ${ }^{7}$, a mis en évidence et analysé cette fonction de maintenant. Selon F. Nef en effet, maintenant en tant qu'opérateur argumentatif peut assumer les trois fonctions suivantes $(1983: 367-370)$ :

- l'annulation d'une implicature conversationnelle de type «si X dit explicitement $p$, alors il sous-entend $q »$; maintenant bloque donc l'obtention de $q$ à partir de $p$ :

(15) Cet homme s'est enrichi très rapidement. Maintenant est-il malhonnête ? (ex. de F. Nef)

(16) Cet écrivain a du succès ; maintenant, a-t-il du talent ? (id.)

- l'annulation d'une implication conditionnelle; maintenant suspend la prémisse, annule le repérage fictif préalablement proposé pour ramener au monde réel ${ }^{8}$ :

(17) S’il venait, je serais heureux ; maintenant, hélas, il ne viendra pas (id.)

- la rectification illocutionnaire, dans laquelle maintenant substitue à un acte de parole initial doté d'un certain type illocutionnaire un acte de parole second doté d'un autre type illocutionnaire ; dans l'ex. 18, le locuteur semble d'abord souscrire à l'affirmation de Pierre, puis rectifie ce positionnement :

(18) Oui, Pierre affirme que Paul a volé dans la caisse. Maintenant, moi, je n’en crois pas un mot (id.)

Et F. Nef de conclure :

7 Merci à M. Vuillaume de m'avoir communiqué cette thèse dactylographiée qui n’a pas été publiée, semble-t-il.

8 Il est à noter qu'il s'agit d'un emploi fréquent et bien répertorié dans les grammaires de l'adverbe «maintenant » en grec ancien, quand, accompagné d’un connecteur légèrement adversatif, il suit une hypothétique : vvv $\delta \varepsilon$ signale alors le retour au réel après le repérage fictif. 
«Dans tous les cas [...], maintenant a pour fonction d'empêcher une proposition de s'intégrer à l'ensemble des présuppositions de l'allocutaire, en ramenant rigidement à ce qui est le cas, contre ce qui peut être dérivé par implicature, implication conditionnelle ou principe illocutionnaire. » (1983 : 370).

On peut donc résumer ses analyses ainsi :

$>\quad$ maintenant temporel : 1 ) indexe $p$ sur $t_{0} 2$ ) suggère par implication négative que ce qui est le cas maintenant ne l'était pas l'instant d'avant ;

$>$ maintenant non-temporel : 1) indexe $p$ sur $\mathrm{m}_{0}$ 2) remet en question une présupposition ou une implicature $^{9}$;

$>\quad$ les deux emplois de maintenant ont donc en commun : 1) la rigidité indexicale 2) le renvoi à l’actualité 3) la négation d'un élément antérieur.

\subsection{Analyse critique}

Dans l'ensemble les observations de F. Nef nous paraissent pertinentes et il faut apprécier à sa juste valeur l'effort fait pour unifier les emplois temporels et non-temporels de l'adverbe sous un signifié commun consistant à exprimer le retour à l'actualité avec une implication négative rétroactive plus ou moins marquée.

Cependant on peut faire ici trois remarques.

- Tout d'abord, les différences établies entre les trois fonctions de ce maintenant argumentatif paraissent largement contextuelles et on aurait peutêtre intérêt, au niveau d'une analyse linguistique visant à établir la forme schématique abstraite sous-jacente à ces emplois, à les regrouper sous un fonctionnement commun, à savoir la rectification ou l'inversion illocutionnaire. On voit en effet que les exemples donnés pour illustrer l'annulation de l'implicature conversationnelle s'accompagnent aussi d'un changement d'acte illocutoire: on passe d'une assertion affirmative par laquelle le locuteur prend pleinement en charge le contenu de la proposition à une interrogation dubitative par laquelle il manifeste une incertitude. Dans le cas de l'annulation de l'implication conditionnelle (dont l'illustration ne me convainc d'ailleurs qu'à moitié), on passe d'une assertion sur repérage fictif permettant d'exprimer indirectement un souhait à une assertion véhiculant un constat de réalité et accompagné d'un regret explicite (hélas). Par ailleurs,

9 Avec $\mathrm{t}_{0}$ nunc de l'énonciation, origo temporelle incluse dans $\mathrm{m}_{0}$, mode de ce qui est (on reconnaît ici le cadre théorique de R. Martin.) 
tous les exemples de notre corpus confirment la place centrale qu'il convient d'accorder à la modification de l'acte illocutoire - nous y reviendrons.

- En deuxième lieu, on peut se demander s'il convient vraiment d'intégrer au signifié en langue de l'adverbe maintenant l'implication négative sur un élément antérieur. Il semble préférable d'y voir un effet contextuel contrastif soutenu par la position initiale détachée de l’adverbe dans tous ces emplois : cette position implique en effet, c'est bien connu, une focalisation à laquelle le destinataire va vouloir donner sens, or la solution la plus banale pour construire ce sens est bien évidemment d'opposer l'élément sous focus et tout qui se trouve sous sa portée à ce qui le précède immédiatement ou, éventuellement, à un implicite à reconstruire ${ }^{10}$.

- Enfin, on remarquera que F. Nef n'envisage qu'une seule origine énonciative possible pour l'ancrage déictique de maintenant; il s'agit dans tous les cas pour lui de l'origine absolue, $\mathrm{t}_{0}$ coordonnée incluse dans $\mathrm{m}_{0}$. Ce choix oblige à voir dans maintenant un marqueur du retour à l'actualité de l'énonciation, ce qu'il n'est pas toujours (cf. ex. 11). Il faut donc envisager, à côté du repère-origine, la possibilité d'existence de repères fictifs (maintenant + conditionnel) ou de repères translatés (maintenant + imparfait).

Toutes ces remarques remettent finalement en cause assez sensiblement le schéma synthétique que nous avions tiré des analyses de F. Nef. Nous allons donc revenir à notre corpus pour tenter de progresser.

\subsection{Les leçons de notre corpus}

Dans notre corpus, qu'il soit un connecteur métadiscursif ou un opérateur argumentatif, maintenant articule toujours deux actes illocutoires différents, le second venant limiter la portée du premier, voire - carrément - l'inverser, ce qui rapproche alors notre adverbe des connecteurs concessifs. En voici quelques exemples.

- Passage d'une assertion exprimant une certitude à une interrogation exprimant un doute (voir aussi l'ex. 8 et sa glose) :

(19) C'est un éclaircissement bien tardif donné au public. Mais enfin je pense qu'il aura eu un certain retentissement. Maintenant trouvez-vous que ce soit assez et que cela nous mette à l'abri des récidives ? Devons-nous publier une lettre de moi ? (G. Sand, Correspondance)

(20) Mon cher ami, Je suis très touchée de vos éloges, car ils sont très affectueux, et très flattée de vos vers, car ils me semblent très beaux. Je ne m'y connais guère, quoique je les aime beaucoup. Mais ceux-là me

10 De la même façon, un « Moi, je » s'oppose explicitement ou implicitement à un « Toi, tu » ou un « Lui, il » ou à toute autre personne déductible du contexte. 
paraissent pleins d'idées, et la forme en est belle, à coup sûr. Maintenant, est-ce que je mérite tout cela ? Non certainement, mais, si vous le pensez de moi, sans en être vaine, j'en suis reconnaissante. (G. Sand, Correspondance)

(21) Et voilà pourquoi Phileas Fogg commandait au lieu et place du capitaine Speedy, pourquoi enfin l'Henrietta se dirigeait vers Liverpool. Seulement, il était très clair, à voir manœuvrer Mr. Fogg, que Mr. Fogg avait été marin. Maintenant, comment finirait l'aventure, on le saurait plus tard. (J. Verne, Le tour du monde en 80 jours)

(22) [...] cela prouve que non seulement des hommes ont débarqué sur cet îlot, mais encore qu'ils l'ont habité pendant un certain temps. Maintenant, quels étaient ces hommes? Combien étaient-ils ? Combien en reste-t-il ? (J. Verne, L'île mystérieuse)

(23) Vous voilà toutes les deux en tenue nocturne dans vos cuisines avec vos téléphones, tandis que les Hommes dorment furieusement.

Vous : Dis-moi la vérité ! Est-ce que je suis un flop en tant que mère?

Fille Aînée est une fille épatante. Elle ne manifeste aucune surprise de se voir réveillée par une créature hystérique en train de se remettre en cause au milieu de la nuit.

Fille Aînée : Tu es une Mère ! C'est déjà formidable. Maintenant, comment savoir si l'on est une bonne ou une mauvaise mère par les temps qui courent? (N. de Buron, Qui c'est, ce garçon ?)

(24) Cherchant ensuite la correspondance Bourg-Lyon, nous constatâmes que M. Darzac avait mis sa dépêche à Bourg une minute avant le départ pour Lyon du train de neuf heures vingt-neuf. Or, ce train arrive à Lyon à dix heures trente-trois, alors que le train de $\mathrm{M}$. Stangerson arrivait à Lyon à dix heures trente-quatre. Après le détour par Bourg et leur stationnement à Bourg, M. et Mme Darzac avaient pu, avaient dû rejoindre M. Stangerson à Lyon, où ils étaient une minute avant lui ! Maintenant, quel drame les avait ainsi rejetés de leur route? Nous ne pouvions que nous livrer aux plus tristes hypothèses qui avaient toutes pour base, hélas ! [...] (G. Leroux, Le parfum de la dame en noir)

(25) Ainsi les phénomènes électrostatiques seraient dus à l'élasticité de l'éther, et les phénomènes électrodynamiques à sa force vive. Maintenant, cette élasticité elle-même devrait-elle s'expliquer, comme le pense Lord Kelvin, par des rotations de très petites parties de fluide ? (H. Poincaré, La Théorie de Maxwell et les oscillations hertziennes)

Dans tous ces exemples, maintenant enchaîne sur le contenu communiqué par l'énoncé précédent, lequel ne se résume pas nécessairement aux contenus sémantique et argumentatif explicites, mais peut reprendre aussi l'acte perlocutoire et les intentions portées par ce dernier. Ainsi, dans l'ex. 24, l'enchaînement peut être glosé de la manière suivante ${ }^{11}$ : «toutes ces

11 Cette glose, comme celle de l'ex. 8, est inspirée des paraphrases de cela dit par C. Rossari, in Rossari et al. (2004 : 114 et sq.). 
déductions minutieuses ont pu vous donner à penser que j’ai reconstitué toute la logique de l'affaire et que j'en possède la clé ; maintenant (= mais à ce stade) je dois vous dire qu'il reste au moins une question en suspens pour laquelle je n’ai que des hypothèses mal fondées ».

- Passage d'une situation repère préconstruite non fictive ( $\mathrm{Sit}_{0}$ ou $\left.\mathrm{Sit}_{1}\right)$ à une situation fictive ou hypothétique (Sit' ${ }_{0}$ ) : voir les ex. 11, 12, 13, 14 ;

Puis il me dit : mais votre article ne sera pas compris. Sur cent personnes qui le liront, à peine deux qui le comprendront... Ici, ils sont enragés contre votre article... et cela tient simplement à une chose, c'est que le sens artiste manque à une infinité de gens, même à des gens d'esprit. Beaucoup de gens ne voient pas. [...]. Maintenant si, avec ce sens artiste, vous travaillez dans une forme artiste, si à l'idée de la forme vous ajoutez la forme de l'idée ... oh! alors, vous n'êtes plus compris du tout... (G. Flaubert, Correspondance)

Dans ce type d'occurrences, maintenant accompagne un changement de repère pour l'énoncé, lequel permet généralement d'ouvrir un nouveau champ de possibles après ce qui pouvait être considéré par le récepteur comme une clôture définitive (ex. 11 : je ne compte pas la voir ; ex. 12 : il n'y a pas de problème; etc.). Seul l'ex. 13 offre une configuration un peu particulière puisqu'il fait passer d'une interrogation-sollicitation orientant vers $p$ (tu vas m'aider) à une concession (ça va) qui représente un abandon de la proposition $p$ au profit de la proposition non- $p$; on a donc affaire à un véritable inverseur argumentatif.

Mais qu'il s’agisse de rectifier une inférence de la communication, de rouvrir le champ des possibles ou d'inverser radicalement l'orientation argumentative des énoncés précédents, maintenant articule toujours deux actes illocutoires différents au fil d'une progression discursive qui fait la part belle au dialogisme.

\section{Altérité énonciative et dialogisme}

A la lecture des exemples du corpus, on est en effet frappé par la présence systématique des marqueurs d'altérité énonciative dans l'environnement immédiat de maintenant, quelle que soit sa valeur contextuelle exacte: constructions hypothétiques, termes lexicaux relevant du champ de la possibilité (pouvoir, être possible), expressions alternatives (qu'il y ait ceci ou [qu'il y ait] cela), interrogations délibératives laissant totalement ouverte la réponse envisageable, etc. En voici trois illustrations :

(27) Je venais ce matin vous voir avec ce singe de Stanislas, qui me précédait de quelques pas, lorsqu'en arrivant là, dit-il en montrant la porte du boudoir, il prétend vous avoir vue avec M. de Rubempré dans une situation qui ne lui 
permettait pas d'entrer, il est revenu sur moi tout effaré en m'entraînant, sans me laisser le temps de me reconnaître; et nous étions à Beaulieu, quand il me dit la raison de sa retraite. Si je l'avais connue, je n'aurais pas bougé de chez vous, afin d'éclaircir cette affaire à votre avantage ; mais revenir chez vous après en être sorti ne prouvait plus rien. Maintenant, que Stanislas ait vu de travers ou qu'il ait raison, il doit avoir tort. (H. de Balzac, Les illusions perdues)

(28) Si à son retour d'Angleterre, vous le rencontriez, il ne faudrait pas me contredire, car pour ne pas le désoler et le brouiller avec moi, il faut que j'aie dit la vérité. C'était la vérité, il y a deux jours, et je tiens à vous dire que je n'ai pas fait d'erreur. Maintenant, qu'il y ait prime double ou prime simple, vous savez que je ne marchande pas et que la question n'est pas là pour moi. (G. Sand, Correspondance)

Si vous descendez pas tout de suite aux barrages, je vous fous de force dans la barque et vous crèverez d'insolation avant d'y arriver. Maintenant si vous préférez, vous pouvez déguerpir, mais alors, en vitesse. (M. Duras, Un barrage contre le Pacifique)

\subsection{Cadre théorique}

Sans doute convient-il ici de préciser ce que j’entends exactement par altérité énonciative, notion empruntée à $\mathrm{A}$. Culioli ${ }^{12}$. Tout énoncé véhicule une représentation construite par le sujet énonciateur et relevant d'un certain domaine notionnel ; en tant que telle, soit cette représentation renvoie à l'intérieur du domaine, c'est-à-dire à une situation ou une occurrence identifiable à l'ensemble des situations ou occurrences qui ont la propriété $p$ définitoire du domaine : par exemple « un individu qui a travaillé avec les Allemands est un salaud » (ex. 12) ; soit elle renvoie à l'extérieur du domaine, c'est-à-dire une situation ou une occurrence identifiable à l'ensemble des situations ou occurrences qui n’ont pas la propriété $p$ définitoire du domaine ou qui ont prioritairement des propriétés autres que $p$ : «tel individu particulier n'est pas un salaud - ou, du moins, offre des propriétés autres qui occultent celle-ci »; soit encore - et c'est ici que l'altérité joue à plein - l'énoncé construit une représentation complexe où aucun des termes de l'alternative $p / p^{\prime}$ n'est éliminé ${ }^{13}$. Une telle représentation peut être construite d'emblée; elle suppose alors un positionnement énonciatif hors-champ en quelque sorte, le choix d'un lieu d’observation " décroché », au point de bifurcation permettant d'envisager à la fois l'intérieur et l'extérieur du domaine ${ }^{14}$; tel est le positionnement 
exprimé, par exemple, dans l'énoncé il est possible qu'il vienne par lequel venir et ne pas venir sont envisagés à parts égales. Mais la représentation complexe peut aussi être le résultat d'un cheminement qui fait passer de IE à I (intérieur du domaine), puis à $\mathrm{E}$ (extérieur du domaine); en somme, il s'agit du cheminement qui permet de parcourir les deux branches de l'alternative et dont l'issue est variable selon les contextes (maintien à égalité des deux possibles, pondération en faveur de l'un sans que soit totalement éliminé l'autre, par ex. avec il est probable, stabilisation totale sur l'un au détriment complet de l'autre, par ex. dans l'extérieur du domaine avec la négation). Un tel parcours peut être assumé par un seul et même énonciateur dont le point de vue évolue alors au fil de son discours, produisant par làmême des effets d'auto-dialogisme, ou peut être affecté à une succession de points de vue pris en charge par des énonciateurs différents, donnant ainsi lieu à du dialogisme interlocutif, éventuellement interdiscursif ${ }^{15 .}$ En voici deux exemples :

(30) [auto-dialogisme d'un linguiste réfléchissant] Je peux, bien sûr, envisager une opposition à trois termes ; maintenant, si je veux rester dans le cadre d'une stricte opposition structuraliste binaire, alors il me faut [...]. (ex. fabriqué)

(31) [dialogisme interlocutif] Il y a aussi des règles fixes pour traduire le langage euclidien dans le langage non-euclidien, ou s'il n'y en a pas, on pourrait en faire. Et si même, il n'y avait ni interprète, ni dictionnaire, si les Allemands et les Français, après avoir vécu des siècles dans des mondes séparés, se trouvaient tout à coup en contact, croit-on qu'il n'y aurait rien de commun entre la science des livres allemands, et celle des livres français ? Les Français et les Allemands finiraient certainement par s'entendre [...]. Mais, dira-t-on sans doute, les Français seraient capables de comprendre les Allemands même sans avoir appris l'allemand, mais c'est parce qu'il reste entre les Français et les Allemands quelque chose de commun, puisque les uns et les autres sont des hommes. On arriverait encore à s'entendre avec nos non-euclidiens hypothétiques, bien qu'ils ne soient plus des hommes, parce qu'ils conserveraient encore quelque chose d'humain. Mais en tout cas un minimum d'humanité est nécessaire. C'est possible, mais j'observerai d'abord que ce peu d'humanité qui resterait chez les non-euclidiens, suffirait non seulement pour qu'on pût traduire un peu de leur langage, mais pour qu'on pût traduire tout leur langage. Maintenant, qu'il faille un minimum, c'est ce que je concède; (R. Poincaré, La valeur de la science)

\subsection{Retour sur maintenant}


Mon hypothèse centrale est que l'adverbe maintenant a, en tout contexte, pour fonction fondamentale de marquer l'étape essentielle de ce cheminement énonciatif, celle où l'on bifurque d'une branche de l'alternative à l'autre ; il articule donc les deux représentations contraires, d'où son rôle de connecteur d'une part, d'opérateur argumentatif d'autre part ; mais ce sont là des fonctions dérivées ${ }^{16}$. Sa fonction principale est bien d'instaurer le nouveau repère énonciatif qui, dans la gestion de l'altérité, va permettre de passer d'une représentation à une autre, de signaler le moment précis de la bascule et du changement de point de vue ${ }^{17}$. Ce repère est construit en discours et n'est pas nécessairement identifié à - ni même dérivé de $-\mathrm{t}_{0}$.

Cette hypothèse explicative me paraît présenter quelques avantages.

Le premier est bien sûr de conserver l'unité du marqueur en dépit de la variabilité de ses emplois; il s'agit bien dans tous les cas d'un adverbe temporel signalant l'émergence d'une nouvelle actualité discursive et permettant l'ancrage de l'énoncé sur celle-ci. En même temps se trouve éliminé l'éventuel problème de la redondance énonciative : on pourrait se demander en effet à quoi sert d'assortir un énoncé métadiscursif du type je dois dire, je le concède, etc. de l'adverbe maintenant, alors que tout énoncé de ce type réfère par définition au fameux hic et nunc de l'énonciation. Mais la redondance disparaît si l'on considère que la fonction de maintenant est de jalonner le temps énonciatif du dire et de construire un nouveau moment discursif ouvrant sur un nouveau point de vue (d'où la fréquence des changements de postures illocutionnaires précédemment signalée). Ce maintenant est en quelque sorte un antidote à l'effacement énonciatif ${ }^{18}$.

Le deuxième avantage est de rendre compte, dans un cadre théorique précis et par le biais d'une opération énonciative dûment répertoriée, d'un effet de sens régulièrement constaté et décrit en des termes variés, à savoir l'implication négative sur le segment antérieur de l'énoncé (F. Nef), la prise de conscience subjective nouvelle (L. de Saussure) ou encore la fonction de « rupteur temporel » (M. Wilmet).

Enfin le dernier avantage de cette analyse est d'intégrer maintenant à un ensemble de phénomènes convergents. Tout d'abord, l'évolution de la fonction temporelle de l'adverbe vers la fonction argumentative en lien étroit avec l'émergence d'un point de vue a été très bien analysée par A. Rabatel dans un article qui associe dans un même type de comportement maintenant,

16 T. Nyan (1991 : 163) note à juste titre que, contrairement à mais, maintenant ne stabilise pas l'argumentation en faveur du second énoncé ni de la conclusion non-r ; il ouvre la possibilité de prendre en compte un nouvel élément et laisse à l'interlocuteur le soin de prendre position. 
cependant, puis et alors, avec en miroir le comportement inverse de mais ${ }^{19}$; dans ce cadre, les adverbes temporels étudiés (auxquels on pourrait ajouter au demeurant) sont estimés être des « co-embrayeurs du point de vue ». Par ailleurs, au-delà des adverbes temporels, on note que nombreuses sont les occurrences où un autre déictique que maintenant articule la progression énonciative qui fait passer de l'intérieur du domaine à son extérieur et exhibe ainsi l'altérité des points de vue successivement présentés par le sujet énonciateur :

(32) Moi, ce que j'en dis, c'est parce que c'est le beau-père (J. Giono, Colline)

(33) Vous savez, ce que j'en dis, c'est pour causer. Moi, je m'en bats l'œil. (G. Chepfer, Saynètes, Paysanneries 2)

En (32) comme en (33), maintenant pourrait aisément soit commuter avec le pronom personnel (moi ; vous savez), soit s'y adjoindre ${ }^{20}$.

\section{Conclusion}

On conclura par cette citation d'A. Culioli (1990 : 102-103) qui, tirée d'un développement sur la négation, paraît pourtant tout à fait adaptée à notre propos :

«On voit que tout marqueur fournit l'histoire d'une construction par laquelle on engendre une bifurcation, un repère énonciatif, l'assignation de positions subjectives, la sélection, à partir d'une telle position origine, d'une représentation dans un couple pondéré de représentations (...). En d'autres termes, il n’y a pas de marqueur isolé, il n’y a pas de marqueur sans la trace mémorisée de sa genèse, il n'y a pas de marqueur (ou d'agencement de marqueurs) qui ne soit issu de l'ajustement de deux représentations complémentaires appartenant au même domaine d'une catégorie notionnelle ; tout objet (méta)linguistique recèle une altérité constitutive. »

\section{Références}

Achard, P. (1992). Entre deixis et anaphore: le renvoi du contexte en situation. Les opérateurs alors et maintenant en français, in: M.A. Morel ; L. Danon-Boileau, (éds.), La deixis, Paris: PUF (« Linguistique nouvelle »), 583-592.

19 Cf. Rabatel (2001).

20 Ces exemples imitant fortement l'oral, il faudrait aussi pouvoir prendre en compte les faits intonatifs qui accompagnent la bascule de point de vue. 
Adam, J.-M. (1987). Enonciation et textualité. Les connecteurs : l'argumentation dans le texte, Cahiers du Département des Langues et des Sciences du Langage, Université de Lausanne, $\mathrm{n}^{\circ} 4$.

Bres, J. ; Haillet, P.-P. ; Mellet, S. ; Nølke, H. ; Rosier, L. ; (éds) ; (2005). Dialogisme et polyphonie: approches linguistiques, Bruxelles: De Boeck / Duculot.

Culioli, A. (1990). Pour une linguistique de l'énonciation. Opérations et représentations, Paris / Gap : Ophrys, tome 1.

Jaubert, A. (1993). A propos, que je te dise, ... et autres 'pertinentiseurs', in Actes du XXème Congrès international de Linguistique et Philologie Romanes, Tome I, section 1 : La phrase, Tübingen / Basel : Francke, 317-328.

Jollin-Bertocchi, S. (2003). La polyvalence de l'adverbe maintenant, L'Information Grammaticale 97 : 26-30.

Nef, F. (1980). Maintenant ${ }_{1}$ et maintenant $_{2}$ : sémantique et pragmatique de maintenant temporel et non-temporel, in : J. David ; R. Martin, (éds.), La notion d'aspect, Metz : Centre d'analyse syntaxique de l’Université de Metz («Recherches linguistique » t. V, diffusé par Klincksieck), 145-166.

Nef, F. (1983). Contribution à l'étude des relations entre logique et linguistique: la description de la deixis temporelle du français moderne. Thèse dactylographiée, Paris : Paris IV-Sorbonne.

Nef, F. (1986). Sémantique de la référence temporelle en français moderne, Bern : Peter Lang.

Péroz, P. (1998). Maintenant, il y avait du temps. Invariance des opérations et instabilité des origines, Le Gré des Langues 13 : 80-111.

Rabatel, A. (2001). La valeur délibérative des connecteurs et marqueurs temporels mais, cependant, maintenant, alors et puis dans l'embrayage du point de vue, Romanische Forschungen 113, 2 : 154-170.

Rossari, C. ; Beaulieu-Mansson, A. ; Cojocariu, C. ; Razgouliaeva, A. (2004). Autour des connecteurs. Réflexions sur l'énonciation et la portée, Bern : Peter Lang.

Victorri, B. ; Fuchs, C. (1996). La polysémie : construction dynamique du sens, Paris : Hermès. 